\title{
Naturalistic study of the antipsychotic medication review service at the Maudsley Hospital
}

\author{
AIMS AND METHOD \\ To evaluate the effectiveness of the \\ antipsychotic medication review \\ service (AMRS) at the Maudsley \\ Hospital. Patient notes were analysed \\ from the AMRS and estimates of \\ Global Assessment Scale (GAS) scores \\ were made from entries in the notes. \\ Data on hospital admissions before \\ and during attendance at the AMRS \\ were obtained from the trust-wide \\ computerised patient administration \\ system.
}

\author{
RESULTS \\ A statistically significant improve- \\ ment in GAS scores was seen for \\ patients who stayed in contact with \\ the AMRS. Patients who did not \\ respond to the first atypical drug \\ often made a good response to an \\ alternative atypical antipsychotic. \\ Patients attending the AMRS had \\ fewer hospital admissions than they \\ did before attendance, although this \\ was not statistically significant.
}

\begin{abstract}
CLINICAL IMPLICATIONS
Although more expensive on a doseby-dose rate, atypical antipsychotics may be cost effective by improving compliance and reducing the number of relapses and hospital admissions. Specialised services with frequent patient contact can be effective in preventing relapse and improving global function.
\end{abstract}

The antipsychotic medication review service (AMRS) is a specialised service run at the Maudsley Hospital (south London). It was developed initially as a 'test-bed' for atypical antipsychotic drugs so that expertise with these compounds could be gained in order to inform practice throughout the hospital. The AMRS has a broad remit and currently provides drug regime assessment as well as outpatient-based treatment for patients with psychosis.

These patients may be partially responsive to or intolerant of typical antipsychotics. The AMRS also accepts referrals of patients with first-episode psychosis. The service aims to minimise drug polytherapy, extrapyramidal symptoms and hyperprolactinaemia while maximising effectiveness through the use of atypical antipsychotic medication as monotherapy. Adjunctive drug treatments and augmentation strategies are not used routinely in the AMRS and formal psychological or psychosocial interventions are not a regular part of treatment, although informal motivational techniques are used. Treatment in the AMRS is underpinned by its emphasis on the formation of an effective therapeutic relationship: efforts are made to share as much information as is available and patients are encouraged to take an active role in choosing their treatment. Patients unresponsive to treatment in the AMRS are referred to the local clozapine clinic.

The study was designed to compare treatment within the AMRS with that previously received by patients. The aims of the study were to verify the usefulness of atypical antipsychotics in a naturalistic setting as well as to evaluate a dedicated clinic as secondline treatment for patients who do not improve with standard out-patient care.

\section{Method}

\section{Sample collection}

The clinical notes were obtained for all patients who had been referred to the AMRS since its inception in
September 1997, up to and including July 2000. The hospital's computer-based patient administration system, which provides data about number and length of treatment episodes, including admissions for all patients within the South London and Maudsley Trust, was searched to provide admission statistics for all patients referred to the AMRS from within sector.

\section{Clinical data collection}

The reason for referral, the date of the referral and the length of time seen in clinic were recorded, as was the diagnosis of each patient and whether there was concurrent drug or alcohol misuse. The medication that each patient was on before being admitted to clinic and the change in their medication while they were being seen in clinic were also noted.

\section{Instruments}

A psychiatric rater (J.S.) applied the Global Assessment Scale (GAS) to the notes as described by Dill et al (1989) for the first assessment in the AMRS and at monthly intervals for the first 6 months. For the second 6 months, ratings were applied at 3-monthly intervals and then 6 -monthly up to 2 years. The outcome was recorded for each patient. Outcomes included recovery and referral back to local carers (for patients who made a marked recovery on the new treatment), assessment for medication review (for patients who were referred for review of medication only), continuing treatment (for patients in the clinic who made a response to medication but were still being followed up), non-compliance (with medication or with attendance) and deterioration despite compliance (including patients referred on to the clozapine clinic). The notes were evaluated retrospectively and ratings were independent of the clinical team. 


\section{Statistics}

original papers
Data were analysed for change in GAS after 1 month, 1 year and 18 months. Tests of significance were made, where appropriate, using student's $t$-test, the $\chi^{2}$ test and Fisher's exact test. Software package SPSS version 8.0 was used to determine $95 \% \mathrm{Cls}$, to calculate significance and to provide a graphical representation of results.

\section{Results}

\section{Patient sample}

Sixty-three patients had been referred to the service since its inception. Of these, 38 were male and 25 were female; 44 were referred from within the South London and Maudsley Hospitals Trust, 14 were referred from a neighbouring hospital trust and 5 were national referrals. Five patients did not attend following referral.

\section{Clinical characteristics}

Of those who attended, the diagnosis was recorded as schizophrenia in 49 cases, with 6 cases of psychosis (unspecified) and 3 of schizoaffective disorder. The reason for referral was fairly evenly spread between treatment resistance $(n=24,41.4 \%)$, treatment intolerance $(n=20,34.5 \%)$ and first-episode psychosis $(n=14$, $24.1 \%)$. Nine (15.5\%) patients had concurrent substance misuse.

\section{Attendance}

The mean duration of contact with the clinic was 200 days ( $n=58, s . d .=245$ ). The mean frequency of attendance (including those referred for review only) was one appointment per 15.7 days of contact with the clinic $(n=58$, s.d. $=11.7)$.

A total of $13 / 58$ (22\%) patients left the clinic before formal discharge. The mean time of contact with the clinic for this group was 47 days (s.d. $=42.1$ ).

\section{Medication on referral}

The antipsychotic medication regimes on referral are summarised in Fig. 1. Eighty-six per cent of patients were being treated with an antipsychotic at referral $(31 \%$ on atypical antipsychotics, $41 \%$ on typical antipsychotics and $14 \%$ on polytherapy). Of those on an atypical antipsychotic at referral, eight were referred because of only having made a partial response, four were intolerant of their medication, five had a first-episode psychosis that was partially treated and one was non-compliant with medication.

\section{Antipsychotic polytherapy}

Eight (14\%) patients were being treated with more than one antipsychotic at referral but after starting in the clinic and following the crossover period to atypical antipsychotics all but one were on monotherapy. This difference was statistically significant $\left(n=58 ; \chi^{2}\right.$ test, $P=0.015$; Fisher's exact one-tailed test, $P=0.015$ ).

\section{Anticholinergic use}

Fifteen (26\%) patients were being treated with oral anticholinergics when referred to the clinic but none continued on these while in the clinic $\left(n=58 ; \chi^{2}\right.$ test, $P<0.001)$

\section{Medication started in clinic}

A total of $47 / 58(81 \%)$ patients were treated with an atypical antipsychotic in the clinic. Twenty $(42 \%)$ patients were started on quetiapine (mean dose $460 \mathrm{mg}$; s.d. 175.2). Eighteen (38\%) were started on olanzapine (mean dose $14.4 \mathrm{mg}$, s.d. 5.72), five (11\%) on sertindole (mean dose $15.8 \mathrm{mg}$, s.d. 5.22 ) and four (9\%) on risperidone (mean dose $3 \mathrm{mg}$, s.d. 1.15).

The mean time in clinic before starting on an atypical antipsychotic (or being changed to a new atypical) was 16 days (s.d. 31.0). The mean period of time for which the two antipsychotic drugs were overlapped was 7 days (s.d. 27.3).

\section{Switching between atypical antipsychotic drugs}

Twenty-one patients were swapped from one atypical antipsychotic to another while in the AMRS. This was due either to non-response or intolerance of the first atypical tried. The outcome for these cases was that nine cases recovered allowing referral back to their home team, five cases continued in contact with the clinic with good response to the new medication, one deteriorated on the new medication and six were non-compliant with medication or clinic attendance.

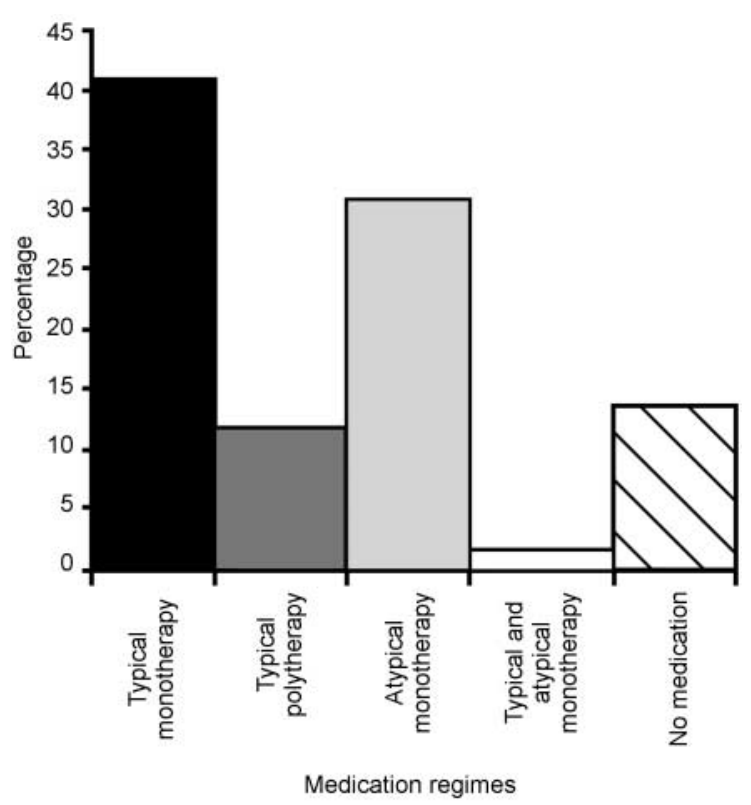

Fig. 1 Medication regimes at referral. 


\section{Patients attending clinic who did not receive an atypical antipsychotic}

A total of $11 / 58$ patients were not commenced on an atypical antipsychotic medication while attending the clinic. The majority of these (7/11) attended for the purpose of a second opinion and subsequently returned to their local teams. The other four patients discharged themselves before being started on an atypical antipsychotic.

\section{Admission data}

The patient administration system covers data on patient admissions exclusively from the South London and Maudsley Trust and so analysis of admission before and during contact with the clinic was limited to this group.

Out of 44 patients referred from South London and Maudsley, four patients did not attend the clinic and so were excluded from the analysis and a further two patients had missing data. Of the remaining group 1 of 38 patients was an in-patient for 18 days while under followup by the clinic. In comparison, 5 of the 38 patients were admitted (a total of 182 in-patient days) in the same period of time before their attendance at the clinic ( $n=38$; paired sample $t$-test, $P=0.11$; Fisher's one-tailed exact test, $P=0.087$ ).

\section{Rating scores}

There was a statistically significant improvement in GAS scores. The improvement was significant after 1 month, increasing from 45 to $50(n=45, P<0.001)$, and this improvement continued for the time that the patients were followed in the clinic. Over 6 months the average GAS score improved from 44 to $58(n=21, P<0.001)$, and over 12 months the average GAS score improved from 41.4 to $61.2(n=13, P<0.001)$. Even though there are limited data on patients who stayed in the clinic longer than a year, because many patients were formally discharged from the clinic by this time, the change in GAS score is statistically significant for 18 months, rising from 43 to $56(n=7, P=0.021)$.

If data on the patients who self-discharged are included in the analysis of the clinic's effect on the GAS by carrying forward their last recorded GAS score (Fig. 2), the improvement is from 45.1 at time 0 to 52.9 at 6 months $(n=58, P<0.001)$. When analysed separately, it is found that the group of patients who self-discharged had a mean GAS score at first contact with the clinic of 46.3 $(n=13$, s.d. $=9.27)$ and that this had not changed significantly at their last contact $(46.5, n=13$, s.d. $=10.0$, $P=0.874$ )

\section{Outcome}

Seventeen patients $(27 \%)$ referred to the clinic recovered and were referred back to their own team for follow-up. Sixteen $(25.4 \%)$ were non-compliant with treatment and $11(17.5 \%)$ were still under monitoring by the clinic. Of the remaining 19 patients, 13 (20.6\%) were referred for medication review only, five ( $8 \%)$ did not attend the clinic

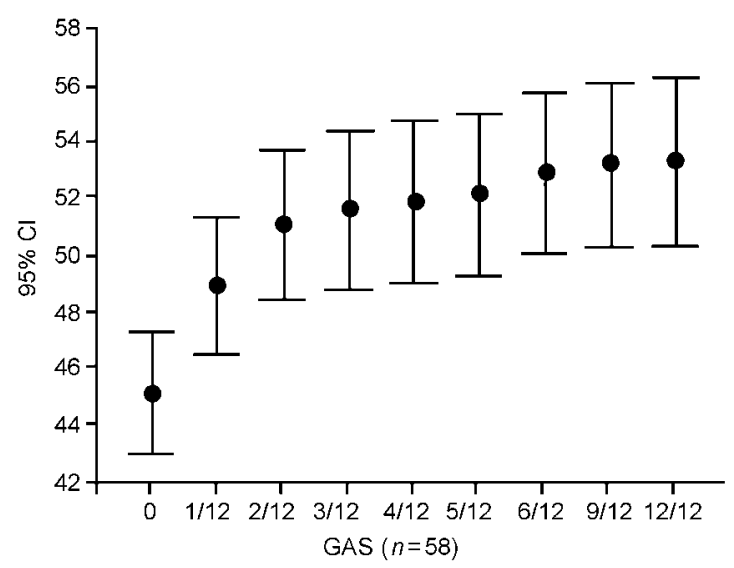

original papers

Fig. 2 Change in Global Assessment Scale (GAS) scores with time, with last observation carried forward for 12 months $(n=58)$.

and one (2\%) deteriorated despite full compliance with treatment (Fig. 3). Substance misuse did not have a significant effect on outcome.

\section{Conclusions}

We have performed a retrospective audit of a novel service providing medication review and atypical antipsychotic treatment for patients with psychosis.

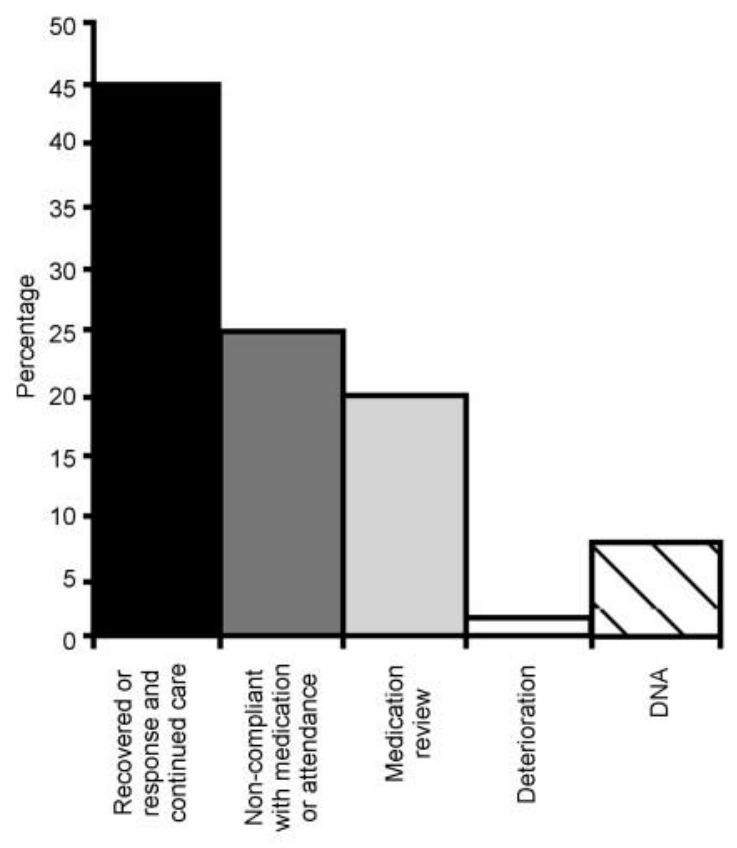

Category

Fig. 3 Outcome showing percentage of cases falling within each category $(\mathrm{n}=58)$.

Recovered, marked improvement and referral back to own team; response and continued care, those patients who were still attending the clinic regularly at the time of the study; non-compliant, non-compliance with continued attendance at the clinic, as well as non-compliance with medication; medication review, those patients who attended the clinic in order to assess their medication regime to provide advice to their own team; deterioration, patients who deteriorated despite apparent compliance with treatment; DNA, patients who were referred to the service but failed to attend any appointments. 
7

original

papers
Patients who remained in the clinic showed symptomatic improvement, as measured by the GAS. This improvement was maintained for the duration of their contact with the clinic (up to 2 years). Patients who did not respond to the first atypical drug often made a good response to an alternative atypical antipsychotic. In many cases this led to almost complete recovery, allowing referral back to their local carers.

Only one patient who was compliant with attendance and medication at the clinic failed to improve and required referral to the local clozapine clinic. This suggests that the atypical antipsychotics other than clozapine are an effective treatment for the majority of patients with psychosis, including those refractory to treatment with other typical or atypical antipsychotics. It is unclear whether improvement was due solely to the medication used or to some other effect from attendance at the AMRS. It may be that attendance at the AMRS led to increased compliance with medication, possibly through making patients feel more empowered in their choice of treatment, but further work is required to clarify this.

This study has shown that atypical antipsychotic drugs used as monotherapy are an effective treatment for psychosis in patients at various stages of illness. The use of atypicals in the AMRS allowed the discontinuation of anticholinergic medication, the use of which can have a negative effect on cognitive function (Borison, 1996; Mizusawa, 1998). Although more expensive on a doseby-dose rate, atypical antipsychotics may be cost effective by improving compliance and reducing the number of relapses and hospital admissions. Specialised services such as the AMRS can be a feasible and effective way of maintaining improvement and preventing relapse in patients with psychosis.

A relatively high proportion of patients became noncompliant with medication or failed to attend the clinic after initial assessment. This is not a new finding for patients with psychiatric illness and is not specific to the AMRS (Killaspy et al, 2000). The non-compliance rate for the AMRS after initial attendance, although high (25\%), compared favourably with data suggesting a $40 \%$ rate of non-attendance at follow-up in a general psychiatric clinic (Killaspy et al, 2000) and with drop-out rates in clinical trials of $36-50 \%$ on a variety of atypical medications (Geddes et al, 2000). Factors that may have led to lower drop-out rates than clinical trials or routine adult general psychiatric settings include systematic and formal assessment of patients, with high-quality dialogue between staff and patients concerning choices of medication, and the continuity of involvement of relatively senior psychiatric staff in treatment and follow-up. Both carers and patients were given time to discuss concerns over medication.

The findings of this study are in keeping with previous work, which has demonstrated the benefits of atypical antipsychotics over typical antipsychotics when used in closely controlled but less naturalistic settings (Stanniland \& Taylor, 2000; Worrel et al, 2000). It is hoped that these data will aid further service development and will assist in defining elements of the AMRS that are transferable to standard community mental health team or depot clinic settings.

\section{Acknowledgements}

The authors wish to thank Drs Hugh Jones, Shubalade Smith, Rodrigo Bressan, Valeria Bigliani and Caroline Stephenson for their assistance in the clinic.

\section{Declaration of interest}

D.T. has received unrelated consultancy fees from all manufacturers of atypical antipsychotics. L.P. has received unrelated and unrestricted charitable grants and lecture and consultancy fees from AstraZeneca, Eli Lilly, Novartis, Janssen Pharmaceuticals, Synthelabo and GlaxoSmithKline. She is also a Medical Research Council Senior Clinical Research Fellow.

\section{References}

DILL, D. L., EISEN, S.V. \& GROB, M. C. (1989) Validity of record ratings of the Global Assessment Scale. Comprehensive Psychiatry, 30(4), 320-323.

BORISON, R. L. (1996) The role of cognition in the risk-benefit and safety analysis of antipsychotic medication. Acta Psychiatrica

Scandinavica Supplement, 389, 5-11.

GEDDES, J., FREEMANTLE, N. HARRISON, P., et al (2000) Atypica antipsychotics in the treatment of schizophrenia: a systematic overview and meta-regression analysis. BM 321, 1371-1376.

KILLASPY H. BANERJEE, S., KING, M., et al (2000) Prospective controlled study of psychiatric out-patient non-attendance. Characteristics and outcomes. British Journal of Psychiatry, 176, 160-165.

MIZUSAWA, H. (1998) Anticholinergic drugs and cognitive functions. Interna Medicine, 37(6), 514-518.

STANNILAND, C. \& TAYLOR, D. (2000) Tolerability of atypical antipsychotics. Drug Safety, 22(3), 195-214.

WORREL, J. A., MARKEN, P. A. BECKMAN, S. E., et al (2000) Atypical antipsychotic agents: a critical review. American Journal of Health Systems and Pharmacy, 57(3), 238-255.

James Stone Senior House Officer, Maudsley Hospital, Ruth Ohlsen Research Nurse Specialist, Institute of Psychiatry, David Taylor Chief Pharmacist, South London and Maudsley NHS Trust, * Lyn Pilowsky Reader in and Head of Section Neurochemical Imaging and Psychiatry, Institute of Psychiatry, London SE5 8AF (e-mail: I.pilowsky@iop.kcl.ac.uk) 